\title{
Medical Image of the Week: Pancreatic Abscess
}

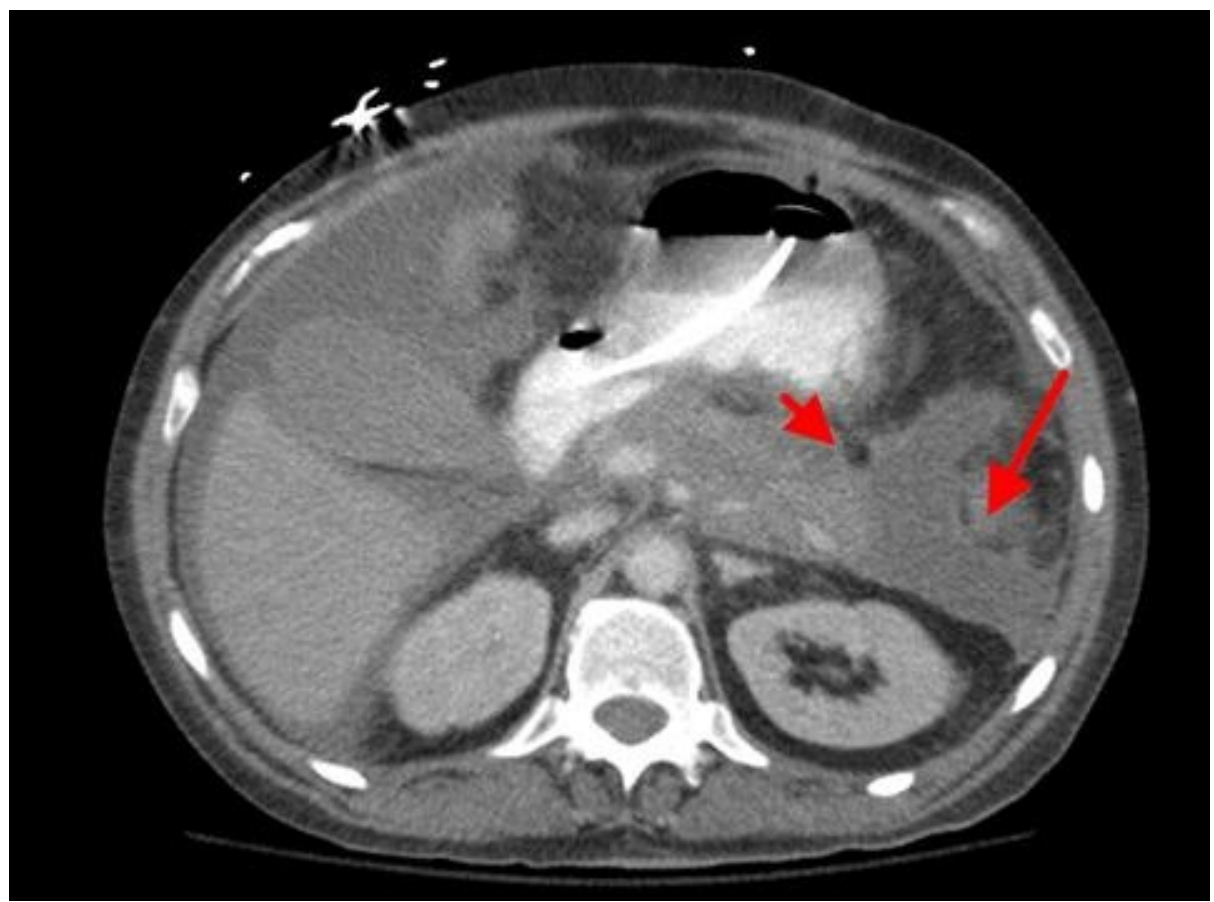

Figure 1. CT scan of the abdomen showing fluid collections (arrows).

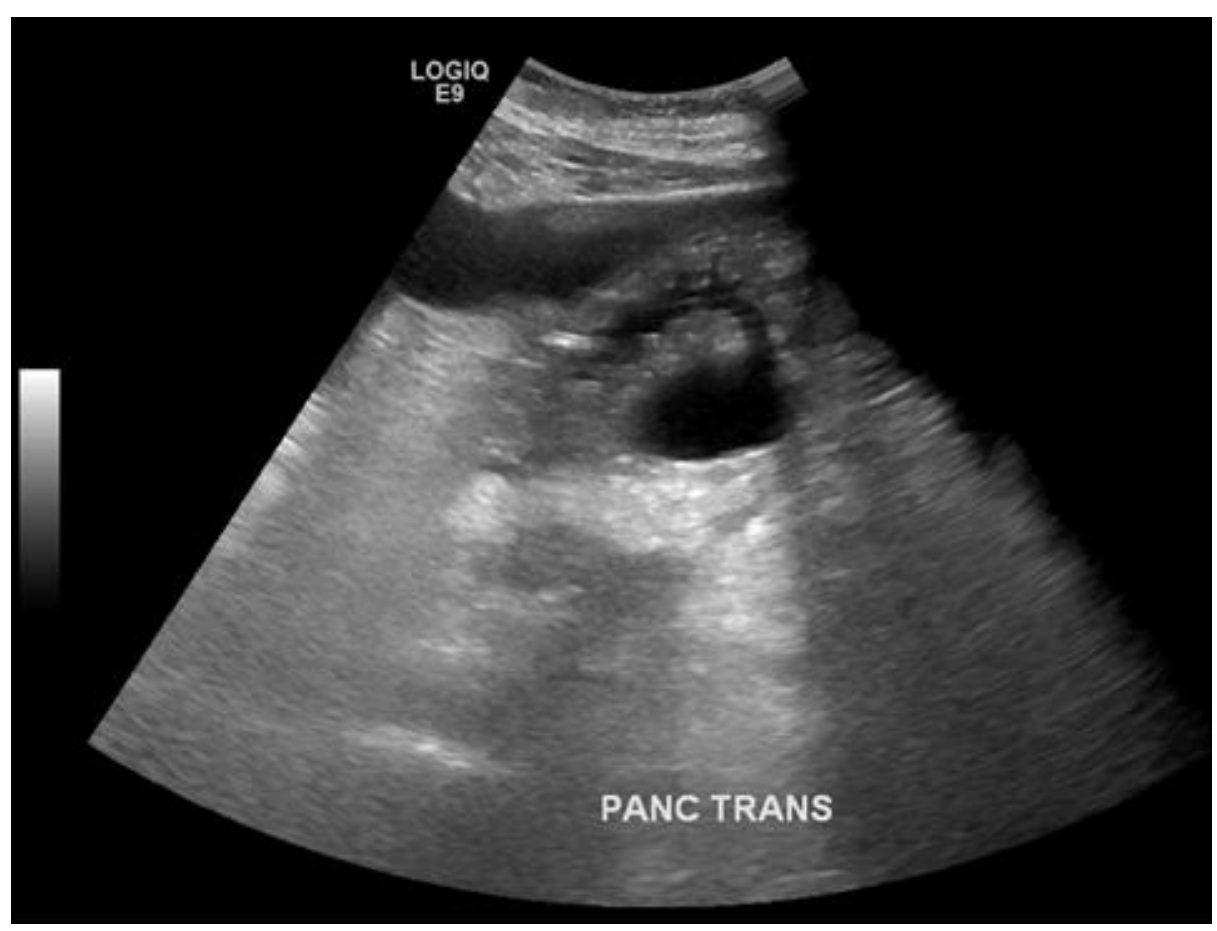

Figure 2. Ultrasound of the abdomen showing a distended pancreatic duct, which communicates to an inferior fluid collection, likely a pseudo-cyst. 


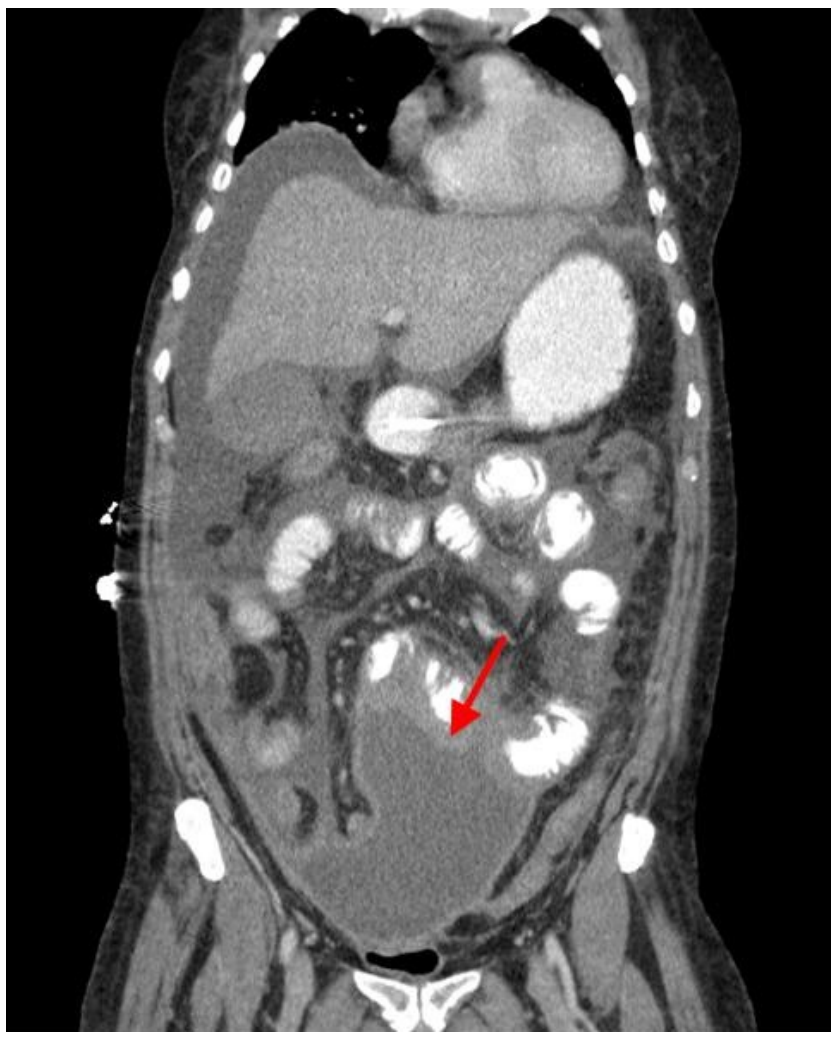

Figure 3. Coronal CT of the abdomen and pelvis showing pelvic abscess (arrow).

A 55 year old woman, with a history of alcohol abuse and necrotizing pancreatitis, was readmitted for worsening abdominal pain and acute respiratory failure. A CT scan of the abdomen and pelvis showed an atrophic pancreas and multiple fluid collections. Along the inferior surface of the pancreas, there is a fluid collection with an evolving loculated rim, which is asymmetric, the larger component measure $2.9 \mathrm{~cm} \times 4.7 \mathrm{~cm}$ (Figure 1, large arrow). Anterior to the body of the pancreas, there is an additional $2.2 \mathrm{~cm} \times 2.4 \mathrm{~cm}$ with evolving loculated rim (Figure 1, short arrow), both compatible with a pseudocyst. Ultrasound of the abdomen showed a distended pancreatic duct that communicates to the smaller fluid collection (Figure 2). Coronal CT of the abdomen and pelvis showed a 12.4 $\mathrm{cm}$ pelvic abscess (Figure 3 ). CT guided drainage of the pelvic abscess was performed with positive culture of the fluid for $E$. coli. She developed secondary peritonitis and had a successful exploratory laparotomy.

Carmen Luraschi-Monjagatta MD, Mohammed Alzoubaidi MD, Elizabeth Ulliman MD,

Department of Pulmonary, Allergy, Sleep and Critical Care.

Internal Medicine, South Campus.

University of Arizona

Tucson, Arizona 